\title{
The Interconnection of Medicals and Distribution of Services for Doctors Using the Principle of Petri Net
}

\author{
Prof. Lokanath Sarangi ${ }^{1}$, Prof. Subhrata Mishra ${ }^{2}, \quad$ Dr. C R Panda ${ }^{3}$, \\ ${ }^{1}$ Asst.Prof. \& Head ECE, NMIET, BBSR \\ ${ }^{2}$ Asst. Prof Dept. AEI, MIET, BBSR, \\ ${ }^{3}$ Prof. \& Head EEE, KEC, BBSR
}

\begin{abstract}
:
The Petri net model presented in this paper focuses on the modeling and the simulation of the medicals bidding behavior and the service system dispatch by the doctors. Where Petri net models capture its stochastic and distributed characteristics. A hybrid multilayer Petri net market simulator, which combines the modeling of physical, owes and information owes, is proposed. The base layer is the physical layer, where the service transmission networks are modeled. On top of it are information layers modeling information owes which schedule the physical owes via discrete tokens. In between, there is an interface layer coded as programs and functioning as a control agent. Colored Petri nets are used to simulate the information layer, in which the uncertainties presented in the decision making process during the bidding and the parallel behaviors of the bidders are modeled. An extension of continuous Petri nets, called a Variable Arc Weighting Petri net (VAWPN), is introduced to simulate the physical layer, in which vector tokens are used to match the information owes to the physical owes and distribution factors are used to obey the physical laws of the service. The market simulator consists of three major modules: the ISO module, the Medical Organization (MO) module and the Load module. The Independent System Operator (ISO) module simulates the priority-based service distribution process following a Location based Marginal Pricing (LMP) scheme. In addition to allowing the medical bids to be functions of price, the algorithm also accepts load bids as functions of price. Bidding strategies are developed for various types of medicals, based on which a medical organization module is developed to provide medical bids to the ISO module. With the price feedback from the ISO module, the MO module can allow the bidders to adjust their bids or switch bidding strategies. The Time Sharing Entity (TSE) model develops load bids to the ISO model. TSE are considered to be either an aggregated demand or price sensitive block bids depending on the situation studied. Impacts of medical bidding strategies on the market clearing prices (MCP) are discussed based on the simulation results. Future research directions in this area are also addressed.
\end{abstract}

Key words: VAWPN, TSE, MCP, MO, DAM, RTM \& LMP

\section{1: Introduction}

This paper reviews the existing modeling methods for medical service systems and the topics relevant to the research. The motivation for the deregulated service in medical system in details, the reason that Petri nets were selected as our approach to model the scheduling of doctors problem of an open access hospital management. The objective of this paper is to build a multi layer Petri net model to model and simulate the deregulated medical services. A new type of Petri net model called Variable Arc Weighting Petri net (VAWPN) is used to model the physical layer, which consists of the medical systems physical infrastructure like equipments, no of beds, different field expertise doctors and the location of the medical. The colored Petri net models are suitable to model the medicals and the doctors. The Time Sharing Entities (TSEs), the Medical Organization (MO), and the market regulator forms the Independent System Operator (ISO). The ISO model follows the Location based Marginal Pricing scheme and prepares both medical and doctors bills based on a price merit demand. It cares the time bound capacity into consideration and can be used to study the congestion rejects on the market clearing prices (MCPs). Bidding strategies were implemented in the MO model which provides bills to the ISO model. The medical cost curves and service availability strategies are derived. Risk hedging issues are addressed by accounting the availability of medicals and the degrading value of equipments. Billing strategies for medicals with limited capacities in small medical units are also analyzed. Price feedback incenses on the patient's billing strategies are studied based on the simulation. Each participant medical is driven by an incentive of achieving minimized profit for themselves choosing only those billing strategies that will achieve maximum profit with their medical units operated within constraints.

\section{1: Motivation}

A major component of any service industry is a transport system that delivers services to the consumers. Examples of such systems include airlines, packages, freight, and medical services. There are many commonalities between these systems such as pricing and congestion. It would be highly desirable that a common modeling approach can be applied to these systems serving 
IOSR Journal of Engineering

Apr. 2012, Vol. 2(4) pp: 848-853

diverse customers. This paper focuses on the modeling of deregulated medical services and discusses a new modeling approach for hospital management systems. Although the modeling techniques developed in this paper are intended for medical service with little modifications, they can be extended to many other service systems as well, for example, air traffic systems [4]. The deregulated medical service is more than ever determined by distributed decision making and driven by discrete events, which are hard to simulate and model in traditional ways. A regulated system has the following characteristics:

\section{(i) Medicals are dependent and their is a single optimization objective function}

An optimal economic service schedule is established based on the assumption that a utility will serve the time of doctors according to their needs of its own customers at minimum cost. Interconnected operations and duty interchange agreements are the result of inter utility arrangements with all the medicals sharing common interests.

\section{(ii) Centralized decision making}

A centralized duty distribution center controls the scheduling and dispatch of its own system. A carry on scheduling function is performed by each medical rather than self scheduling. Therefore, all the functions are coordinated to meet a single economic objective for the utility that owns them.

\section{(iii) Deterministic}

The scheduling and service problem is a deterministic optimization problem due to the fact that the cost curve of each medical unit is used to form an objective function, which is the total cost of the service, together with constraints due to all the physical constraints. There are no human behaviors such as economic or physical that can withhold the service to patient. Cost curves are known for each type of medical with some uncertainties caused by the variations of types of problems to patient. The medical service is bid based and three time-sequential service markets are established:

(i) The bilateral trade market (BTM)

(ii) The day ahead market (DAM)

(iii) The real time market (RTM)

The Independent System Operator (ISO) provides an equal access to distribute services by doctors for all interlinked medicals. Medical organizations compete in the DAM and RTM markets to sell their services. Medicals providing service in different entities setup forecasted treatment demands in the DAM and the difference between the real demand and the committed DA time of doctors in the RT system.

The changes that have brought to the health service are profound:

\section{(i) Multiple optimization objective functions}

The economic objectives of the market participants are not identical. For medical a minimization of the total service time of doctors cost and a maximization of non overlapping of schedule are the objectives. The goal is minimization of payment. Minimizing total service cost will not, in general, result in minimization of total payment from patient. The uniform price rule for each type of desires is used because the market clearing price is set by the most expensive medical running an expensive local hospitals may indeed lower the overall service cost. But it results in higher market clearing price which causes more service payment. For ISO, the market regulator who carries out a priority-based service, where the medical bids are received and evaluated in a merit order based on both price and operational constraints. The goal is to get a near optimal solution within network physical constraints, while maintaining system reliability and security.

\section{(ii) Distributed decision making}

The ISO contributes the service time of individual doctor, but it can not dictate the number of patient present and their requirements. Operations and planning of medical service are now decentralized with demand forces. However, their behaviors are not totally independent. In response to each other's bidding strategy, market participants perform strategic bidding. The ISO contributes the gaming and sometimes tacit collusion all of which further complicates the situation.

\section{(ii) Uncertainties}

In a deregulated market, the information available to medical services are limited and regulated, or received with time delay [4]. In addition, a decision made by one participant may impact the overall system dispatch. These difficulties are compounded by the underlying uncertainty in the Institution from where the doctors are qualified. Unscheduled outages of medicals and tactics used by other personal clinic of doctors. Consequently, a market participant needs to hedge its supply and demand commitments to reduce potential risks arising from such uncertainties. 
IOSR Journal of Engineering

Apr. 2012, Vol. 2(4) pp: 848-853

\section{(iii) Congestion Management}

Open access to the service system greatly encourages the interchange of time among different doctors. Congestion caused by limited timeline capacities can have a profound impact on salary of different doctors because of the Location based Marginal Pricing (LMP) scheme, which is introduced to differentiate the service cost of busy doctors. These emerging changes call for new modeling methods and new service algorithms to better represent and operate the restructured service system, as well as to provide more insights in its optimal operations.

\section{2: Existing Modeling Methods}

A single integrated medical service system operating both to Doctors and their service time for the patient, the utility could establish between the per hour service charge and the types of service [2] with an objective of minimizing its operating costs while taking into consideration all of the necessary physical, reliability, and economic constraints. A typical cost curve for a steam turbine unit is shown in Figure 1.1a. Usually the cost can be represented by either a quadratic or a cubic function of the service in hours. An incremental cost curve $\mathrm{dC}=\mathrm{dH}$ can be derived accordingly. For a quadratic cost curve, its incremental cost curve will be piece-wise linear as shown in Figure 1.1b. The beginning part and the ending part can be represented by different slopes to relate the different rates of cost changes.

The simplified dispatch problem for $\mathrm{T}$ periods of service and $\mathrm{N}$ medicals can thus be related as

$$
\begin{aligned}
& \min \sum_{t=1}^{T} \sum_{i=1}^{N}\left[\boldsymbol{F i}\left(\boldsymbol{H} \boldsymbol{i}^{t}\right)+C \boldsymbol{s i}, \boldsymbol{t}\right] \boldsymbol{i}_{i}^{t}=\boldsymbol{F}\left(\boldsymbol{H}_{i}^{t}, \boldsymbol{U}_{i}^{t}\right) \\
& U i^{t} H i^{\mathrm{min}} \leq H i^{t} \leq U i^{t} H i^{\mathrm{max}} \text { for } \mathrm{i}=1 \ldots \ldots \mathrm{N} \text { and } \mathrm{t}=1 \ldots \ldots \mathrm{T} \\
& H^{t} \text { service }-\sum_{t=1}^{N} H i^{t} U i^{t}=0, \text { for } \mathrm{t}=1 \ldots \ldots \ldots \mathrm{T}
\end{aligned}
$$

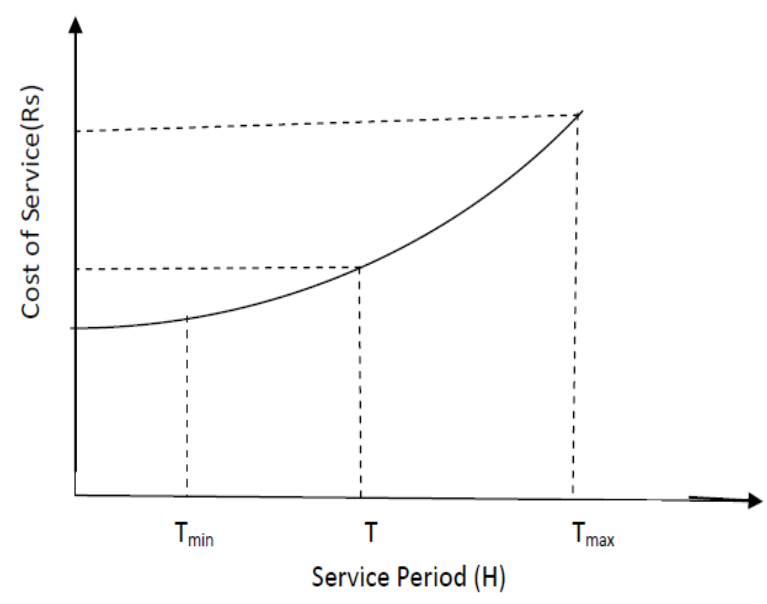

Fig.1.1(

a)

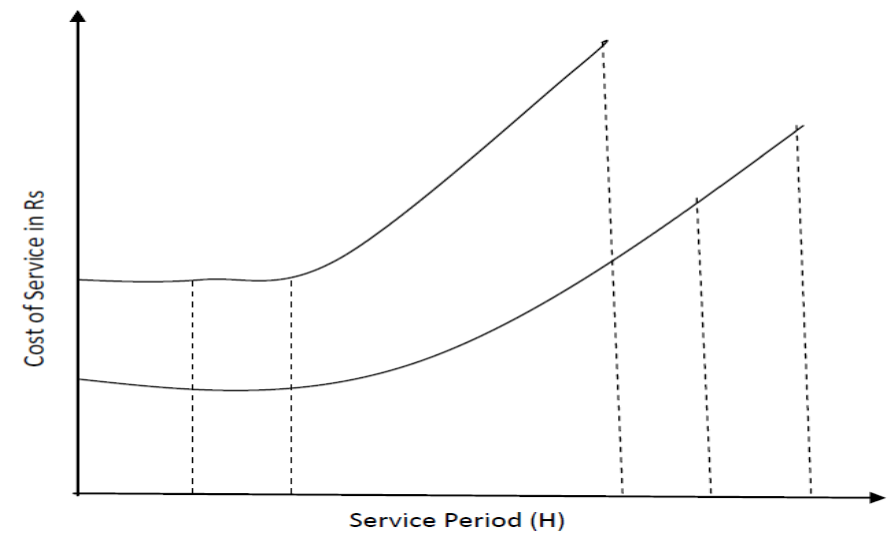

Fig.1.1(b)

Where $\mathrm{Hi}^{\mathrm{t}}=$ the total hours of service during period $\mathrm{t}$.

$\mathrm{H}^{\mathrm{t}}$ service $=$ the total patient during period $\mathrm{t}$.

$\mathrm{C}_{\mathrm{S}} \mathrm{i}, \mathrm{t}=$ The start cast of service for I unit of patient in time $\mathrm{t}$.

$\mathrm{Ui}^{\mathrm{t}}=0$, if unit I no. of patient are absent during t period.

$\mathrm{Ui}^{\mathrm{t}}=1$, if unit i no. of patient are absent during t period.

$\mathrm{Ui}^{\mathrm{t}}=1$, if unit I no. of patient are present during t period.

$\mathrm{F}\left(\mathrm{Hi}^{\mathrm{t}}, \mathrm{Ui}^{\mathrm{t}}\right)$ is the cost of the medical $\mathrm{T}$ is the total time of service.

$\mathrm{N}$ total no. of medicals

The Lagrangian function can be formed as [2]

$\mathrm{L}(\mathrm{H}, \mathrm{U}, \lambda)=\mathrm{F}\left(\mathrm{Hi}^{\mathrm{t}}, \mathrm{Ui}^{\mathrm{t}}\right)+\sum_{i=1}^{T} \lambda^{t}\left(H^{t}\right.$ load $\left.-\sum_{i=1}^{N} H i^{t} U i^{t}\right)$ 
IOSR Journal of Engineering

Apr. 2012, Vol. 2(4) pp: 848-853

Where $\mathrm{q}(\lambda)=\min _{\mathrm{Hi}^{\mathrm{t}}, \mathrm{Ui}^{\mathrm{t}}} \mathrm{L}[\mathrm{H}, \mathrm{U}, \lambda]$
and $\mathrm{q}^{*}(\lambda)=\max _{\lambda^{\mathrm{t}}} \mathrm{q}(\lambda)$

Again $\mathrm{L}(\mathrm{H}, \mathrm{U}, \lambda)$ is the Lagrange function and $\lambda$ is the Lagrange multiplier.

Using optimization principle in two steps [7], the value of $\lambda^{t}$ which moves $q(\lambda)$ towards a large

Value and assuming the value of $\lambda^{t}$ as calculated as fixed, then the minimum of $L$ is calculated by adjusting the values of $\mathrm{H}^{\mathrm{t}}$ and $\mathrm{U}^{\mathrm{t}}$.

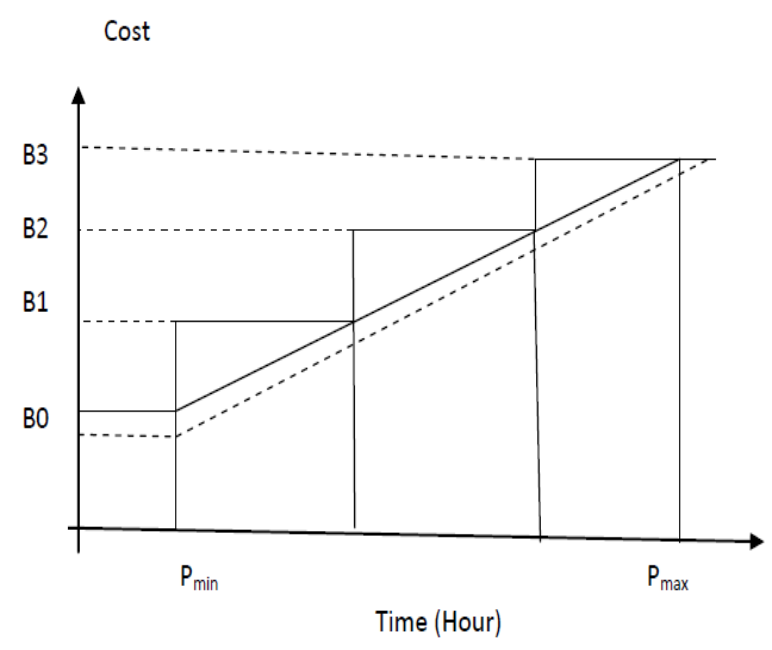

Fig.1.2(a)

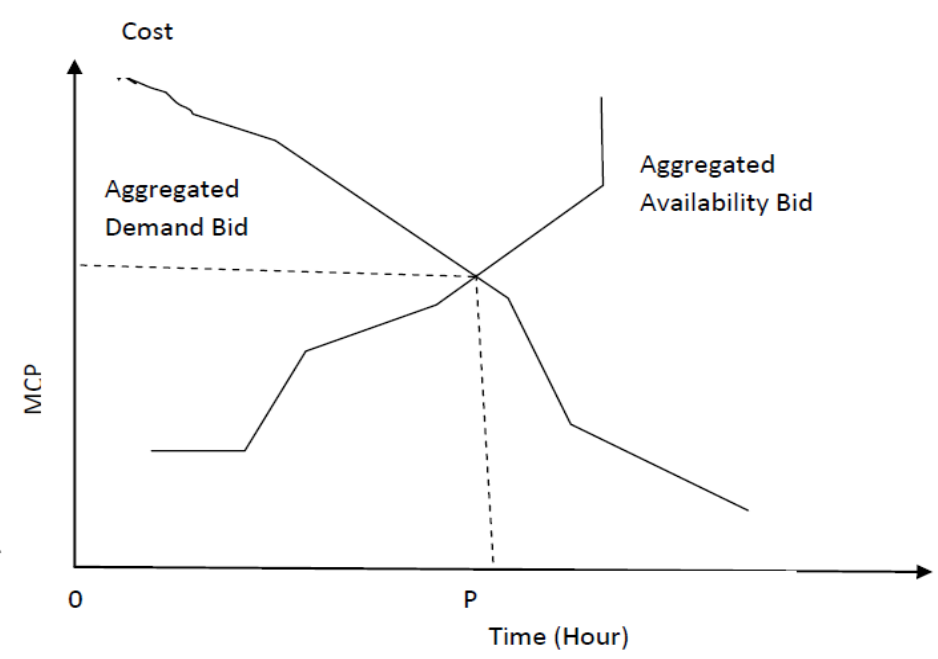

Fig.1.2(b)

In a regulated system, a central coordinator with all the cast curves of services $\mathrm{F}\left(\mathrm{Hi}^{\mathrm{t}}, \mathrm{Ui}^{\mathrm{t}}\right.$ ) available performs the optimization represented in figure (1.2). The goal of the optimization is to minimize the service cast imposed on the patient. In a deregulated service system the medical association set up a piece-wise linear and monotonically increasing service charge as mentioned in figure (1.2a) per hour basis. According to the agreement between government \& medical association there is a "demand bid curve". Based on the demand and availability of doctors. The request of which the "Market clearing price" (MCP) for each hour is shown in figure (1.2b).

The time to be allotted for each medical by a doctor is then determined based on the individual. The time to be schedule for each doctor is then determined based on the individual busy curve of doctors \& the MCP. All the time provides will be compensated at the MCP. To fix up the time based payment to doctor the pay curve of other medical \& the time of engagement of each doctor to be uncertainties in the time fixing of emergency cases. The goal of the medical is to provide maximum time of service with fewer prices to patient. Sometimes the cast of service may increase by a doctor when he is being busy by two or more medical at same time. The decision making process of each medical for remuneration fixing may be time varying, which causes uncertainties into the price setting schemes and causes the volatility of the MCP in a deregulated system. Game theories [1], [2] have been used to investigate the possible price fixing technique. Discrete price fixing are described as price high, price medium, price slow in matrix games and pay off matrices are constructed by enumerating all possible combinations of strategies. Modified unit commitment methods involves efforts to improve Lagrangian relaxation based price \& implementation and time scheduling.

Since the times sharing properties based under mathematical forms. For a medical simulate the hole process they need to have extensive knowledge about different medical, the types service they provide, the doctors attached to the systems and their network model. The basic purpose of this paper is to develop a graphical representation of the time sharing of individual doctors attached to a single medical or more than one medical. 
IOSR Journal of Engineering

Apr. 2012, Vol. 2(4) pp: 848-853

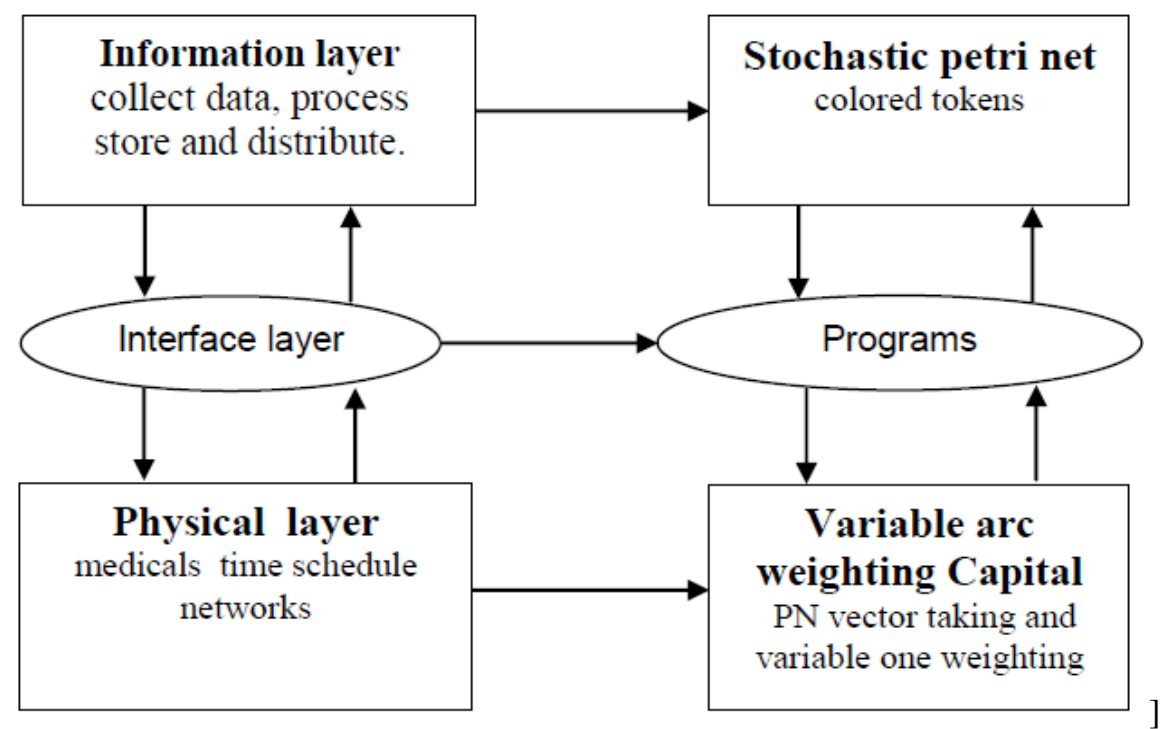

Fig. 1.3: The structure of multilayer PN Model

\section{3: Petri Net Model}

Reflect market rules and reveal the operation mechanism of the doctor's load in times shared to different medicals. Simulate the parallel stochastic and distributed binding behaviors of the medicals interconnected to each other. To provide a medical a tool to develop better binding strategies and evaluate them as a graphical and mathematical tool, Petri nets [ 3], [4] have been successful used in communication protocols and automated manufacturing system, informed which they offer flexibility to simulate discrete systems. Therefore a Petri Net is used as a modeling tool of the deregulated medical services. It is a graphical and mathematical tool for describing studying systems that are characterized has being concurrent, asynchronous, distributed and stochastic it is used as a visual communication aid similar to flowcharts and networks. The simulation dynamic and concurrent activities of a system can be performed by the use of tokens. The setting of algebraic state equation and other mathematical models governing the behavior of the system can be performed using Petri net [5].

The Petri net can be readily used to describe a combined system model if several interacting subsystems. Through token exchange very sub stem can interact with each other. The service model of doctor's is dividing in to three parts like ISO model, medial model and time sharing model. The medical and time sharing model submit medical and time bid to the ISO which then distribute the time of the doctor's according to the importance of the types and suffering of patients. The ISO model is deterministic since every information is available to it. The medical and time sharing model are stochastic to simulate the uncertainties in their decision making process. PNs are logical models derived from the knowledge how the system works and allow the simulation of uncertainties via stochastic transitions [6].

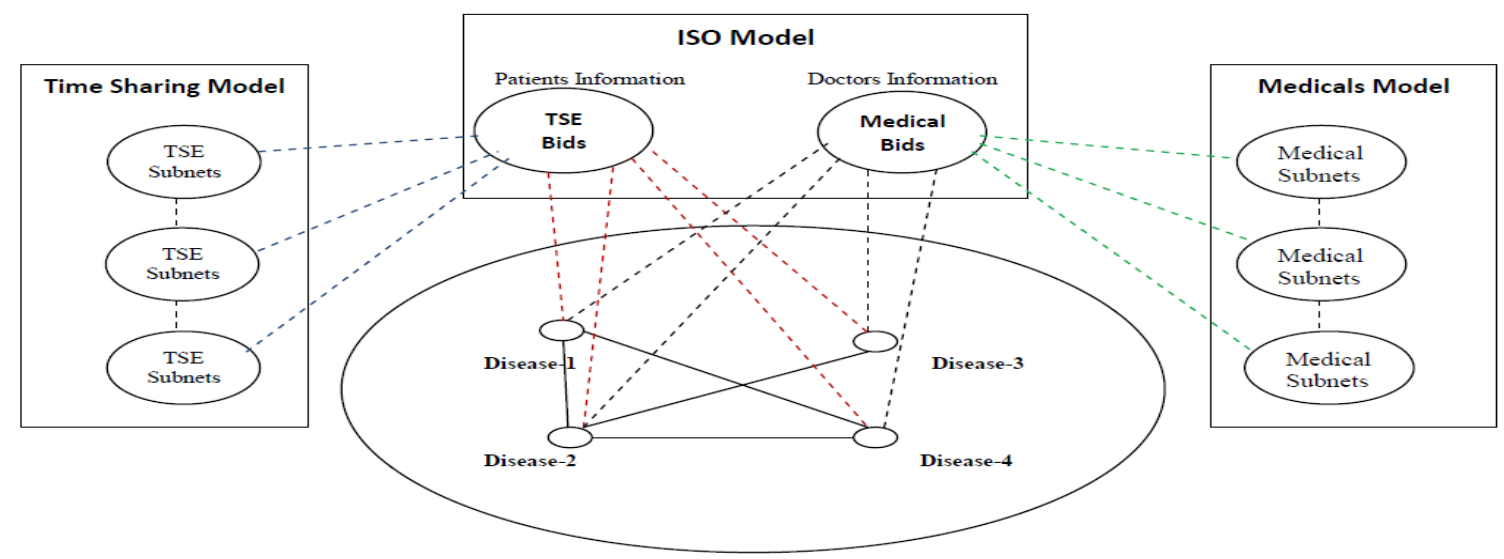

Fig. 1.4: Structure of deregulated medical services 
IOSR Journal of Engineering

Apr. 2012, Vol. 2(4) pp: 848-853

Through PN the location based marginal pricing (LMP) scheme is applied PN model can simulate the simulation like remuneration hike less in one medical to doctors on the service charge hike on less to the patient in the medicals. The discrete event systems can be model in the physical layer which contains continues power-flow under the control of the information layer. The variable arc weighting Petri net (VAWPN) are used to develop the physical layer of the discrete event systems. The interface layer is developed to control operation of the VAMPN and provide communication links between the physical and communication layer.

\section{4: Conclusion}

This paper has introduced the background of ordinary PN, color PN and VAWPN. Initially with one medical the doctors are assigned with their duty and service but threw PN a single doctor can provide his/her service at different medical and different location with variable service charges. A regulated market in the field of medical service can be established with the following characteristics.
(i) Deterministic
(ii) Distributed decision making
(iii) Stochastic
(iv) Congestion management

This model should serve has a simulator to evaluate the impacts of the various biding strategies on the MCP. The interaction of the information flow and physical is also addressed in the building the model. Through graphical representation of the service process it can reflect

(i) Market rules and reveal the coordination mechanism of the doctor's time sharing process.

(ii) Simulate the parallel, stochastic and distributed binding behaviors of the medicals interconnected with each other.

(iii) Provide to the patient a tool to evaluate and develop better strategies.

The above model can be applicable to air traffic, defense, electric power supply, and communication and education system with little modification in the systems.

\section{5: References}

[1] G. Owen, Game Theory, Third ed: Academic Press, 1995.

[2] J. W. Bialek, IGaming the Uniform-Price Spot Market - Quantitative Analysis," IEEE Trans. on Power Systems, vol. 17, pp. 768-773, 2002.

[3] N. Viswanadham and Y. Narahari, Performance Modeling of Automated Manufacturing Systems, Prentice Hall, Inc., New Jersey, 1992.

[4] K. Kanoun, M. Borrel, T. Morteveille, and A. Peytavin, \Availability of CAUTRA, a Subset of the French Air Trafic Control System," IEEE Trans. on Computers, vol. 48, pp. 528-535, 1999.

[5] R. David and H. Alla, IPetri Nets for Modeling of Dynamic Systems - A Survey," Automatica, vol. 30, pp. 175-205, 1994.

[6] A. A. Desrochers, Performance Analysis Using Petri Nets," Journal of Intelligent and Robotics Systems, no. 6, pp. 65-79, 1992.

[7] S. Hao, G. A. Angelidis, H. Singh, and A. D. Papalexopoulos, IConsumer Payment Minimization in Power Pool Auctions," IEEE Trans. on Power Systems, vol. 13, no. 3, pp. 986-991, 1998 